Service social

\title{
La construction identitaire des garçons et la réussite au cégep
}

\author{
Jacques Roy, Josée Bouchard et Marie-Anne Turcotte
}

Volume 58, numéro 1, 2012

URI : https://id.erudit.org/iderudit/1010439ar

DOI : https://doi.org/10.7202/1010439ar

Aller au sommaire du numéro

Éditeur(s)

École de service social de l’Université Laval

ISSN

1708-1734 (numérique)

Découvrir la revue

Citer cet article

Roy, J., Bouchard, J. \& Turcotte, M.-A. (2012). La construction identitaire des garçons et la réussite au cégep. Service social, 58(1), 55-67.

https://doi.org/10.7202/1010439ar

\section{Résumé de l'article}

L'objet de cet article est de rendre compte des éléments tenant spécifiquement à la construction identitaire des garçons dans leur rapport à l'univers scolaire. Le matériel de base de cet article puise à une récente étude que nous avons réalisée en milieu collégial et qui portait sur la réussite scolaire selon le genre. Les principaux constats de l'enquête par questionnaire réalisée auprès d'un échantillon de 611 étudiants répartis dans 27 collèges et d'entrevues de groupe effectuées auprès de 64 cégépiens, sont présentés dans la perspective d’une mise en relation du processus de construction identitaire chez les garçons avec leur parcours scolaire. En point de discussion, deux modèles compréhensifs traduisant l'existence de formes de socialisation différenciées selon le genre des étudiants sont exposés. 


\title{
La construction identitaire des garçons et la réussite au cégep
}

\author{
Jacques Roy \\ Josée Bouchard \\ Marie-Anne Turcotte
}

\section{RÉSUMÉ}

L'objet de cet article est de rendre compte des éléments tenant spécifiquement à la construction identitaire des garçons dans leur rapport à l'univers scolaire. Le matériel de base de cet article puise à une récente étude que nous avons réalisée en milieu collégial et qui portait sur la réussite scolaire selon le genre ${ }^{1}$. Les principaux constats de l'enquête par questionnaire réalisée auprès d'un échantillon de 611 étudiants répartis dans 27 collèges et d'entrevues de groupe effectuées auprès de 64 cégépiens, sont présentés dans la perspective d'une mise en relation du processus de construction identitaire chez les garçons avec leur parcours scolaire. En point de discussion, deux modèles compréhensifs traduisant l'existence de formes de socialisation différenciées selon le genre des étudiants sont exposés.

Mots-clés : Garçons, parcours scolaire, cégep, identité, socialisation, genre, modèles compréhensifs.

\section{Contexte}

Parmi les facteurs conditionnant la réussite scolaire des cégépiens, les facteurs sociaux occupent une part importante, voire déterminante. C'est ainsi que le système de valeurs des étudiants, la conciliation travail et études et certains indicateurs de bien-être personnel, entre autres, sont régulièrement identifiés comme étant des facteurs étroitement associés au rendement et à la persévérance scolaires selon nos travaux (Roy, Bouchard et Turcotte, 2008; Roy, Bouchard et Turcotte, en collaboration avec Tremblay et Fournier, 2010). Selon ces études, les valeurs des cégépiens constituent une sorte d'aimant satellite reliant différents facteurs déterminants de la réussite scolaire, au premier chef la motivation aux études qui représente une variable phare de la réussite selon nos travaux.

Dans la foulée de ce constat, nous nous sommes intéressés plus particulièrement aux valeurs des cégépiens et à certains mécanismes de socialisation selon le genre des étudiants afin d'en dégager des points de convergence et de divergence entre les garçons et les filles. Cette exploration nous a conduits dans un second temps à la question de la construction identitaire des étudiants selon le genre en lien avec leur parcours scolaire.

L'objet de cet article est de rendre compte des éléments tenant spécifiquement à la construction identitaire des garçons dans leur rapport à l'univers scolaire. Le matériel de base

1. Dans ce travail, nous utilisons le concept de genre plutôt que la notion de sexe car ce concept s'apparente aux normes culturelles d'une société qui déterminent des façons d'être, d'agir et de penser ainsi que les sentiments qui apparaissent les plus appropriés pour les femmes et les hommes (Tremblay et al., 2006). 
de cet article puise à une récente étude que nous avons réalisée en milieu collégial et qui portait sur la réussite scolaire selon le genre. II s'agit d'une étude à deux volets : un volet quantitatif portant sur une enquête par questionnaire effectuée auprès de 611 étudiants répartis dans 27 collèges et un volet qualitatif composé d'entrevues de groupe semi-dirigées conduites auprès de 64 collégiens et collégiennes du Cégep de Sainte-Foy (Roy, Bouchard et Turcotte, en collaboration avec Tremblay et Fournier, 2010). Portons dans un premier temps notre regard sur le concept d'identité.

\section{LE CONCEPT D'IDENTITÉ}

L'identité est un concept essentiellement polysémique dont la définition peut varier selon les disciplines, les contextes sociaux et les perspectives théoriques adoptées (Sciolla, 2005; Tremblay et L'Heureux, 2010). Pour notre part, nous nous sommes limités à une perspective sociologique selon laquelle l'identité représente principalement une construction sociale; dans cette perspective, les mécanismes de socialisation par lesquels l'individu intègre les attentes, les normes et les valeurs sociales constitueraient un laboratoire de premier plan pour circonscrire l'identité ainsi construite.

Au-delà d'une diversité apparente quant à la signification accordée à la notion d'identité dans la littérature sociologique, il existe tout de même des points d'ancrage. C'est ainsi que pour Sciolla : «Le terme identité [...] désigne au sens général la capacité d'un individu ou d'un groupe à s'auto-reconnaître et à être reconnu par les autres » (Sciolla, 2005, p. 335). Cette acception du terme d'identité se retrouve chez différents auteurs, dont Bajoit (1997).

C'est donc à travers le regard de l'autre que se tramerait l'identité : « on ne peut développer son identité personnelle sans tenir compte du regard des autres sur soi, de l'interprétation qu'on en fait et de l'image que l'on veut produire devant autrui », comme le rappellent Tremblay et L'Heureux (2010, p. 94). Sur le plan sociologique, ce rapport à l'autre transite nécessairement par un lien social plus général qui le contextualiserait selon la culture et les valeurs sociétales existantes et qui conditionnerait la socialisation de l'individu.

De fait, selon Dubar $(1995,2005)$, la construction des identités individuelles serait intimement liée au processus de socialisation. L'auteur souligne que, selon l'approche culturaliste de la socialisation, cette dernière serait « un modelage des personnalités suivant les traits les plus structurants des cultures jugées essentielles au fonctionnement social » (Dubar, 1995, p. 61). L'identité des personnes serait ainsi modelée par une socialisation particulière ancrée dans une culture commune spécifique.

Un autre trait commun émergeant des écrits sociologiques réside dans l'idée que l'identité se construit plus qu'elle ne s'hérite (Galland, 2007). Elle serait essentiellement une construction en devenir puisant à des sources variées. Pour cet auteur, le concept d'identité offrirait ainsi une avenue fort intéressante pour mieux comprendre sociologiquement les jeunes, pour qui vivre leur jeunesse serait d'abord et avant tout une période d'expérimentation (Galland, 2007). Ce point de vue nous intéresse tout particulièrement d'autant que les cégépiens sont à un âge et dans un lieu - le collège - où la quête identitaire se vit d'une manière intense (Roy, 2011). 
Enfin, l'identité chez l'étudiant serait davantage tributaire de facteurs extérieurs à l'institution d'enseignement; pensons notamment à des modes de socialisation vécus dans différents milieux tels que le travail, le réseau d'amis, le milieu familial, et qui sont source d'influence dans sa construction identitaire. De fait, il existerait une forme de plurisocialisation dans la société mettant en scène des sources variées, parfois même contradictoires, d'influence sur les individus (Lahire, 2004). Et, dans ce contexte, le cégep ne serait qu'un milieu parmi d'autres exerçant son influence sur l'étudiant en concurrence avec d'autres sources émanant de la société en général.

À partir de ces considérations, nous avons défini de la manière suivante la notion d'identité pour les fins du présent article : l'identité est une construction sociale d'auto-reconnaissance chez l'étudiant qui lui permet de se définir par rapport aux autres sur tous les plans de sa vie personnelle et sociale.

En complément à cette définition, précisons que la perspective théorique que nous adoptons s'inspire de l'interactionnisme symbolique et considère l'identité comme une réalité en évolution dans l'espace et le temps; ce serait le résultat d'un processus. Dans cette perspective, l'étudiant est à considérer comme un sujet et comme un acteur, bien sûr conditionné en partie par son milieu, mais pouvant aussi intervenir sur sa propre existence et développer des stratégies dans la construction de son identité. Ainsi, selon Bajoit, « [l']être humain est sujet quand il met en œuvre sa capacité de gérer les tensions entre ce que les autres attendent de lui et ce qu'il attend de lui-même, et qu'il se constitue ainsi en individu et acteur dans la société » (Bajoit, 1997, p. 114). En ce qui a trait plus spécifiquement à l'élaboration de l'identité, Dubar (2005) expose que l'individu tente par diverses stratégies de réconcilier «l'identité pour autrui » et « l'identité pour soi ». Nous considérerons donc ici l'étudiant comme un acteur, à savoir un sujet porteur de sens et qui prend des moyens pour donner une orientation à sa vie. Ses actions, ses attitudes et ses croyances seront analysées de ce point de vue.

Cet éclairage théorique guidera notre analyse de la problématique identitaire des garçons au collège non seulement à partir de l'identification de valeurs et de mécanismes de socialisation qui leur seraient propres, mais aussi à partir des stratégies qu'ils déploient dans leurs parcours personnel et scolaire au cégep.

\section{DIFFÉRENCES SELON LE GENRE}

Cette section puise aux résultats de l'enquête par questionnaire de l'étude de Roy et al., 2010. Rappelons qu'un échantillon aléatoire de 611 étudiants provenant de 27 collèges au Québec a rempli le questionnaire d'enquête.

Afin d'examiner la question identitaire chez les garçons, il nous faut préalablement mettre en évidence ce qui les distinguerait des filles. Le prochain tableau rapporte une première synthèse des différences observées selon le genre: 
TABLEAU 1

Synthèse des différences selon le genre des étudiants

\begin{tabular}{|l|c|}
\hline Comparativement aux filles, les garçons : & valeur du $\mathbf{p}^{2}$ \\
\hline accordent plus d'importance à la compétition. & .000 \\
\hline accordent moins d'importance à la famille. & .000 \\
\hline accordent moins d'importance au respect. & .000 \\
\hline sont moins souvent stressés. & .000 \\
\hline ont moins souvent le sentiment d'être déprimés. & .000 \\
\hline s'arrangent plus souvent seuls en cas de problème. & .003 \\
\hline accordent moins d'importance à l'effort pour réussir. & .003 \\
\hline lisent moins souvent des livres. & .003 \\
\hline considèrent moins que la charge de travail est élevée. & .004 \\
\hline consomment davantage d'alcool. & .009 \\
\hline consacrent moins d'heures à leurs études. & .009 \\
\hline accordent moins d'importance à la réussite des études. & .010 \\
\hline accordent moins d'importance à avoir une famille unie. & .017 \\
\hline font davantage d'activités parascolaires. & .018 \\
\hline accordent moins d'importance à la réussite de leur couple. & .023 \\
\hline accordent moins d'importance au diplôme collégial. & .026 \\
\hline considèrent moins élevé le degré de connaissance des professeurs. & .034 \\
\hline
\end{tabular}

Un premier constat que l'on peut tirer de ce tableau est que parmi les valeurs où l'on observe la plus grande différence entre les filles et les garçons figurent la compétition, plus valorisée chez les garçons, et le respect, la famille et l'effort pour réussir les études, plus valorisés chez les filles.

Un deuxième constat est que, de manière générale, les garçons témoigneraient d'un engagement moins tangible à l'endroit des études que les filles. Ainsi, ils accorderaient généralement un peu moins d'importance que les filles à la réussite scolaire, à la «méritocratie» et au diplôme d'études collégiales. En complément, mentionnons que les garçons rapportent consacrer en moyenne 10 heures par semaine à leurs études, comparativement à 13 chez les filles. Par ailleurs, il importe de souligner à grands traits que cet exercice de comparaison avec les filles ne doit pas occulter le fait que la majorité des garçons sont bien engagés sur le plan scolaire selon les indicateurs retenus dans l'étude. Et ils réussissent bien! Portons notre regard sur la réussite scolaire selon le genre.

2. Le $P$ mesure le degré d'association entre les variables. Pour être significatif, il doit être inférieur à .05. Plus il tend vers .000, plus le degré d'association est élevé. 
Dans notre étude, la réussite scolaire a été appréciée à partir de deux indicateurs, soit le rendement et l'abandon scolaires ${ }^{3}$. Les garçons ont une moyenne scolaire légèrement inférieure à celle des filles (74,0 \% comparativement à 77,8\%); cet écart enregistré entre les garçons et les filles masque le fait que les premiers sont, en proportion, deux fois plus nombreux que les filles à cumuler des notes inférieures à $60 \%$ selon nos résultats, donc à être potentiellement en situation d'échec scolaire.

Également, les garçons semblent moins persévérer que les filles dans leur programme d'études; notamment, ils sont proportionnellement plus nombreux à songer « occasionnellement » ou « sérieusement » à abandonner leurs études (16,7 \% des garçons comparativement à $13,9 \%$ des filles). Encore là, l'écart n'apparaît pas significatif à première vue, mais il faut considérer qu'au terme des études, le taux de diplomation des garçons est généralement inférieur de $12 \%$ à celui des filles (Roy, 2008).

Compte tenu de l'importance du champ des valeurs dans la problématique identitaire, nous avons reproduit dans le tableau 2 une comparaison plus spécifique des valeurs entre les garçons et les filles tout en y jumelant la relation existant entre les valeurs et les indicateurs de la réussite scolaire.

3. Le rendement scolaire a été mesuré à partir des résultats scolaires cumulatifs de l'étudiant dans son programme de formation (automne 2008). Quant à l'abandon scolaire, il fut évalué à partir de la question suivante : «Songes-tu présentement à abandonner tes études au collège? » Trois réponses étaient possibles : 1 . Pas du tout; 2. J'y songe à l'occasion; 3. J'y songe sérieusement. Les étudiants ayant répondu qu'ils y songeaient « à l'occasion » et « sérieusement » ont été regroupés comme groupe potentiellement à risque d'abandon scolaire. 
TABLEAU 2

Répartition procentuelle des garçons et des filles selon le degré d'importance accordée à différents énoncés de valeurs ${ }^{4}$

\begin{tabular}{|c|c|c|c|}
\hline \multirow[t]{2}{*}{ Énoncés de valeurs ${ }^{5}$} & \multicolumn{3}{|c|}{ Degré d'importance ${ }^{6}$} \\
\hline & Garçons & Filles & $\begin{array}{l}\text { Écart garçonsl } \\
\text { filles en } \%\end{array}$ \\
\hline $\begin{array}{l}\text { La famille est une dimension importante } \\
\text { dans ma vie*}\end{array}$ & $61,6 \%$ & $82,4 \%$ & $20,8 \%$ \\
\hline $\begin{array}{l}\text { Respecter l'autre dans sa différence est } \\
\text { important pour moi }\end{array}$ & $63,6 \%$ & $83,5 \%$ & $19,9 \%$ \\
\hline $\begin{array}{l}\text { Il est important d'être compétitif dans la } \\
\text { vie* }\end{array}$ & $30,1 \%$ & $10,7 \%$ & $19,4 \%$ \\
\hline $\begin{array}{l}\text { Je crois à l'importance de l'effort pour } \\
\text { réussir dans mes études }\end{array}$ & $67,5 \%$ & $82,4 \%$ & $14,9 \%$ \\
\hline 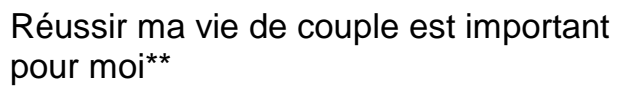 & $63,3 \%$ & $77,2 \%$ & $13,9 \%$ \\
\hline $\begin{array}{l}\text { Bien faire les choses que j'entreprends } \\
\text { est important pour moi }{ }^{\star \star}\end{array}$ & $56,3 \%$ & $70,1 \%$ & $13,8 \%$ \\
\hline $\begin{array}{l}\text { Le diplôme collégial a une signification } \\
\text { pour moi }\end{array}$ & $59,3 \%$ & $72,3 \%$ & $12,9 \%$ \\
\hline $\begin{array}{l}\text { Avoir des projets à long terme est } \\
\text { important pour } \text { moi }^{\star}\end{array}$ & $48,0 \%$ & $58,9 \%$ & $10,9 \%$ \\
\hline
\end{tabular}

Ce tableau illustre ce que nous avons précédemment observé, en insistant cette fois-ci sur les valeurs en lien avec la réussite scolaire qui distingueraient le plus significativement les garçons des filles. Du côté des garçons, la valeur prédominante serait donc l'esprit de compétition - trait de socialisation considéré en général comme plus typiquement masculin qui, selon nos résultats, serait chez eux associé positivement à la réussite scolaire. Chez les filles, les valeurs liées à la sphère de l'intime (famille, vie de couple), à la « méritocratie », de travail bien fait et de projection dans l'avenir (l'importance d'avoir un but, une cible), seraient autant d'aspects d'une socialisation qui prédispose aux études.

Précisons ici que ces valeurs ne sont nullement exclusives selon le genre des étudiants, surtout chez ceux et celles qui réussissent leurs études; dans ce dernier groupe d'étudiants, la convergence des valeurs des garçons et des filles, notamment en ce qui a trait aux études, est même relativement élevée ${ }^{7}$. À l'inverse, l'importance accordée aux études et l'intérêt porté à

4. Le total des répondants varie entre 609 et 611 pour les différents énoncés.

5. Légende : $\left(^{\star}\right)$ facteurs associés à l'abandon scolaire; $\left(^{\star \star}\right)$ facteurs associés à la fois au rendement et à l'abandon scolaires.

6. Le degré d'importance est reflété par le pourcentage d'étudiants ayant répondu être « tout à fait d'accord » avec l'énoncé en question.

7. Notamment, la réussite des études est une valeur considérée comme « très importante » pour la majorité des étudiants, soit $73,7 \%$ des garçons comparativement à $86,4 \%$ des filles. 
celles-ci sont des valeurs qui discriminent davantage les garçons entre ceux qui réussissent bien et ceux qui sont à risque sur le plan de la réussite scolaire; chez les filles, l'écart entre elles est nettement moins prononcé sur ce plan. Ce constat signifie que le rôle joué par ces deux valeurs sur le parcours scolaire (une moindre importance accordée aux études et un moindre intérêt pour la formation chez les garçons à risque) aurait plus d'influence sur le parcours des garçons que sur celui des filles.

En complément à ce dernier constat, soulignons que les garçons sont, en proportion, deux fois plus nombreux que les filles à pointer du doigt le manque d'intérêt pour les études comme motifs d'abandon scolaire, alors que l'inverse s'observe quant aux difficultés scolaires qui sont un motif plus fréquemment souligné par les filles (Roy et al., 2010). Ce constat traduit une logique différente selon le genre qui sera développée plus loin à la faveur de la présentation de deux modèles compréhensifs offrant une perspective d'analyse des différences enregistrées entre les garçons et les filles.

En marge du champ des valeurs, nos analyses ont mis en lumière trois autres traits en lien avec la réussite scolaire qui distinguaient les garçons des filles d'une manière parfois appréciable. Le stress est le premier de ces traits. Selon les résultats de notre étude, deux fois plus de filles que de garçons s'estiment « très stressées » $(20,8 \%$ chez les filles, 10,6 \% chez les garçons); en corollaire, quatre fois plus de garçons que de filles se considèrent comme « aucunement stressés » (3,7 \% chez les filles, 16,3\% chez les garçons).

Le deuxième trait distinctif concerne les réseaux familial et social. De manière générale, chez les garçons, des facteurs liés aux valeurs familiales et au soutien parental aux études semblent agir d'une manière plus sensible sur leur volonté de poursuivre leurs études. De la même manière, le réseau d'amis (présence d'amis désirant abandonner leurs études) pourrait aussi avoir, selon nos résultats, davantage d'influence chez eux que chez les filles au regard de l'abandon scolaire ${ }^{8}$.

Le dernier trait présentant des différences selon le genre relativement à l'abandon scolaire est le bien-être personnel des étudiants. C'est ainsi qu'une autosatisfaction plus faible, le fait de « moins bien se sentir » au collège et une consommation élevée d'alcool seraient en général des facteurs davantage associés à l'abandon scolaire chez les garçons ${ }^{9}$.

Avant de faire place au matériel de nos entrevues réalisées avec des étudiants, mentionnons que le profil des valeurs des étudiants et les traits que nous avons identifiés comme étant associés à la réussite scolaire ne sont pas sans traduire l'existence de mécanismes de socialisation parfois fort différents selon le genre des étudiants. Comme nous l'avons souligné précédemment, ces mécanismes sont à la source de la formation identitaire du jeune garçon ou de la jeune fille. Nous nous en expliquerons plus longuement lorsque nous discuterons des résultats.

8. Chez les filles, ce facteur n'apparaît pas comme étant associé à l'abandon scolaire alors qu'il est présent chez les garçons $(\mathrm{R}=.20, \mathrm{P} \leq .01)$.

9. Pour ces trois facteurs associés à l'abandon scolaire chez les garçons, le coefficient de Pearson (R) varie de .20 à .25 (P P $\leq .01)$. Ces trois facteurs ne figurent pas chez les filles comme associés à l'abandon scolaire. 


\section{IDENTITÉ ET PARCOURS SCOLAIRE}

Dans cette section, nous exposerons les principaux constats découlant des entrevues de groupe réalisées auprès des étudiants en insistant sur ceux qui concernent la construction identitaire en lien avec le parcours scolaire. Tel que mentionné plus haut, 64 étudiants (33 filles, 31 garçons) fréquentant le Cégep de Sainte-Foy et ayant répondu au questionnaire d'enquête ont participé aux entrevues de groupes de discussion focalisée. Ces entrevues ont été réalisées séparément auprès des garçons et des filles pour chacune des trois dimensions à l'étude, soit la relation avec les professeurs, la relation avec les parents et la pratique d'activités parascolaires. Dans le texte qui suit, nous rendons compte des principaux constats selon chacune de ces dimensions. Mais avant, portons notre attention sur certains constats d'ensemble transcendant les dimensions retenues par l'étude.

Ainsi, à la lecture du matériel des entrevues, un premier aspect surgit lorsqu'on regarde la question de l'adaptation aux études collégiales: il semblerait que les garçons aient plus de difficulté sur le plan de la transition secondaire-cégep. Leur désir d'autonomie, qui se traduit souvent par leur volonté de se débrouiller seuls et de ne pas solliciter de l'aide ainsi que leur attitude moins conformiste que celle des filles à l'égard de l'univers scolaire, pourraient expliquer cette situation. Cette autonomie serait alors perçue chez les garçons comme une condition de leur propre développement identitaire.

Bien que l'importance de réussir ses études soit exprimée par un nombre à peu près égal de garçons et de filles, ces dernières, à l'instar de ce que nous avons précédemment constaté dans l'enquête par questionnaire, démontrent un engagement plus affirmé, une intensité plus grande que les premiers. Aussi, les garçons présentent certaines difficultés à se projeter dans l'avenir alors que les filles le feraient plus facilement, accordant davantage d'importance à des projets à plus long terme. De plus, l'attrait du « plaisir immédiat » prendrait plus souvent le pas chez les garçons dans un contexte qui, par ailleurs, commanderait plutôt de s'investir sur le plan scolaire. Les garçons se laisseraient plus facilement «tenter » par des activités ludiques au détriment de leurs études et seraient moins enclins que les filles à se sentir stressés relativement à leurs études.

Première dimension étudiée: la relation professeur-étudiant. Les garçons et les filles s'entendent pour dire qu'elle est importante pour eux. Par contre, les garçons nuancent leur propos en affirmant que d'avoir une bonne relation est un «plus », mais que cela a peu d'influence sur leurs résultats scolaires. Ils considèrent que l'étudiant a sa large part de responsabilité dans sa réussite, qu'il doit se prendre en main, peu importe le professeur. À ce sujet, ils consultent moins leurs professeurs que les filles, préférant se débrouiller seuls ou avec leurs amis. Les garçons semblent plus enclins à développer individuellement leurs modes d'apprentissage et à vouloir contourner les règles déterminées par le monde scolaire. Ce constat serait en lien avec leur désir d'émancipation et leur affirmation identitaire.

Même si les garçons et les filles s'entendent sur des qualités semblables qui sont attribuées à un «bon » professeur, les garçons se distinguent dans leurs attentes. Une attitude pragmatique, le besoin de voir dans du « concret » l'utilité de ce qu'ils font pour garder leur 
motivation les caractérisent davantage. Ils apprécient que leurs professeurs leur fassent expérimenter la matière par eux-mêmes, invoquant que cela leur permet de mieux intégrer les connaissances et se projeter dans un avenir professionnel. Ils sont réceptifs, de fait, à une pédagogie active où est sollicitée une forme de compétition saine qui stimule chez eux le goût de relever le défi et de se dépasser. Ces aspects participeraient plus directement à la manière dont se construit l'identité chez le garçon.

En ce qui concerne la relation avec les parents, deuxième dimension sur laquelle s'est penchée l'étude, la quête identitaire des garçons semble prendre racine dans leur recherche d'autonomie. Ils se situent ainsi dans une logique de détachement des parents. À cet égard, ils soulignent que même si le soutien de leurs parents revêt une importance pour eux, ils ne souhaitent pas que ces derniers se montrent trop insistants à leur endroit dans leur volonté de les aider et dans le fait de les questionner sur leurs choix, leurs études, etc. En effet, même s'ils disent apprécier l'intérêt que leur portent leurs parents et aimer recevoir des encouragements dans leurs études, ils préfèrent néanmoins que ces derniers les laissent aller vers eux en cas de besoin plutôt que de leur démontrer un soutien constant. Ils considèrent avoir moins besoin de se faire rassurer en général de façon continue que les filles, qui manifesteraient, elles, davantage ce besoin. Aspect intéressant à souligner : les garçons ont exprimé que le soutien de leur père était important pour eux alors que les filles n'ont pas évoqué spécifiquement cette dimension.

Les garçons ajoutent que les parents développeraient aussi davantage leur autonomie que celle des filles par la façon dont ils les éduquent en étant moins protecteurs et en insistant sur l'importance de leur « apprendre à se débrouiller ». Certains garçons ont mentionné avoir appris «à la dure », davantage que leurs sœurs qui étaient plus souvent protégées. Le genre aurait donc une influence sur les comportements adoptés par les parents et sur ce qui est attendu des garçons et des filles respectivement. D'ailleurs, les garçons rencontrés ont révélé qu'euxmêmes se considéraient comme « protecteurs » à l'endroit des filles, à l'exemple de ce qu'ils observent dans leur milieu familial.

La quête d'autonomie des garçons peut aussi se manifester dans le choix des sources d'aide lors de moments plus difficiles. En effet, tout comme nous l'avons constaté concernant la relation qu'établissent les garçons avec les professeurs, les répondants (filles comme garçons) ont mentionné que les garçons avaient moins tendance à aller chercher de l'aide directement auprès de leurs parents que les filles. Ils se tourneraient davantage vers les amis ou leur copine lorsqu'ils vivent des difficultés d'ordre personnel.

D'un point de vue plus social, même si les garçons et les filles étaient globalement d'accord sur le fait qu'il s'avère important de «faire quelque chose de sa vie » et de terminer une formation, les participantes ont indiqué que les parents, et même la société, percevraient d'une façon plus positive qu'un garçon se tourne vers des études telles qu'un diplôme d'études professionnelles (DEP) plutôt que des études collégiales ou universitaires. Selon elles, les DEP offrant les meilleures conditions salariales rejoindraient davantage des intérêts masculins, ce qui pourrait expliquer une plus grande acceptation des parents. De plus, parmi les participants, seuls des garçons ont soulevé la possibilité d'utiliser d'autres chemins que les études pour faire 
leur place dans la vie, comme le travail ou l'expérience, alors qu'aucune fille n'a tenu des propos similaires. Ces réflexions ne sont pas sans parenté avec les conclusions de l'étude de Chouinard, Bergeron, Vezeau et Janosz, pour qui les garçons percevraient « l'abandon scolaire comme un choix plus avantageux que ne le perçoivent les filles » (Chouinard et al., 2010, p. 336); parmi les facteurs avancés, les auteurs ont identifié des opportunités d'emploi plus intéressantes pour les garçons non diplômés.

La pratique des activités parascolaires est la dernière dimension examinée. À partir du matériel des entrevues, il est possible d'affirmer que cette pratique aurait une influence sur le développement identitaire des étudiants. En effet, tant les garçons que les filles ont affirmé que ces activités leur permettaient de mieux se connaître personnellement et de s'affirmer davantage comme individu. Elles favoriseraient également le développement de la confiance en soi et de l'estime personnelle. Ces activités leur procureraient une valorisation personnelle et leur permettraient de mieux se définir dans leurs intérêts ainsi que d'identifier certaines forces qu'ils possèdent.

Cependant, une distinction est apparue entre les garçons et les filles. En effet, les garçons ont affirmé être plus individualistes dans la pratique de leurs activités parascolaires alors que les filles ont plutôt insisté sur l'importance de l'appartenance à un groupe. Deux manières différentes de construire son identité! Ainsi, le regard des autres personnes, la reconnaissance personnelle et sociale, auraient une importance accrue pour les filles. Également, celles-ci soulignaient l'intérêt manifeste pour elles de pouvoir compter sur le soutien du groupe d'appartenance associé à leur activité et au partage possible dans ce contexte, alors que les garçons ont moins mis l'accent sur cet élément. Ils ont plutôt parlé de leur fierté personnelle et du dépassement de soi que leur procure la pratique de ces activités.

\section{Discussion}

Un examen des liens qui existent entre l'enquête par questionnaire et les entrevues réalisées auprès d'étudiants sous l'angle de la construction identitaire pendant les études collégiales nous conduit à l'identification de deux modèles compréhensifs distincts selon le genre des étudiants. Deux modèles qui offrent un cadre de réflexion sur des différences observées entre les garçons et les filles. Décrivons chacun de ces modèles.

Du côté des garçons, un modèle d'affirmation de type ludique s'est imposé à la lecture de nos résultats. Expliquons-nous. Dans les entrevues de groupe en particulier, les garçons ont manifesté de différentes façons leur volonté d'être autonomes: autonomes devant les professeurs, devant leurs parents, et valorisant l'esprit de compétition ainsi que la capacité de réaliser seuls une activité parascolaire par exemple. Les garçons carbureraient donc davantage à l'autonomie personnelle et même à une forme d'individualisme, selon des propos recueillis en entrevues de groupe. On a noté aussi chez eux une perspective ludique : ils apprécient que le plaisir, le jeu soient présents dans les démarches d'apprentissage, ils aiment s'éprouver à travers des activités pédagogiques axées, par exemple, sur la recherche de solutions. Leur goût du « concret », leur désir de créer, d'être mis à contribution dans les apprentissages, seraient autant d'éléments renforçant ce volet ludique plus fréquemment observé chez les garçons. 
D'autres, par ailleurs, peuvent exprimer ce côté ludique par une sorte de « dilettantisme », selon l'expression de Rivière et Jacques (2002), à l'égard de leurs études.

Chez les filles, le modèle de l'engagement de type conformiste reproduirait bien ce que nous avons constaté, à savoir un engagement plus intégré et soutenu chez les filles concernant leur cheminement scolaire. Ce dernier se maillerait davantage à leurs propres aspirations personnelles à travers un mode de socialisation où est recherchée la conformité aux exigences scolaires.

Ces modèles évoquent un « idéal-type », soit une représentation abstraite à l'état pur d'un groupe d'individus permettant de saisir le réel à travers une représentation bipolaire (Cherkaoui, 1990). À partir de pôles extrêmes, on pourrait donc déduire ici des conduites propres aux garçons et aux filles en tant que groupes distincts. C'est dans ce contexte que nous proposons ces deux modèles compréhensifs (affirmation de type ludique chez les garçons et engagement de type conformiste chez les filles) comme cadre interprétatif de nos résultats.

Ces deux modèles ne sont pas sans traduire un cadre de construction identitaire distinct chez les garçons et les filles qui reflète des mécanismes de socialisation différents selon le genre des étudiants. Par exemple, la valorisation de l'esprit de compétition chez les garçons ou les mécanismes de collaboration et d'entraide qui sont plus répandus chez les filles. À leur façon, ces deux exemples de socialisation différenciée peuvent contribuer à la construction d'identités sur des bases différentes. Ainsi, en passant en revue les principaux mécanismes de socialisation qui prévalent selon le genre, on reproduit un cadre dans lequel émergent des trajectoires identitaires différentes chez les garçons et les filles.

\section{ConClusion}

L'examen du parcours scolaire des étudiants au cégep, de leurs attitudes, de leurs valeurs, a mis en évidence certaines différences selon le genre. Ces différences, comme nous venons de le souligner plus haut, sont largement tributaires de mécanismes de socialisation distincts selon le genre. La construction identitaire des garçons, comme celle des filles, puiserait largement à ces mécanismes.

Tout au long de notre étude, un fil d'Ariane s'est imposé du côté des garçons : leur quête d'autonomie. Celle-ci serait nettement plus présente et affirmée chez eux que chez les filles. Aussi, elle expliquerait bon nombre d'attitudes et de comportements des garçons à l'égard du monde scolaire. 
Plus globalement, notre recherche a mis en évidence l'existence de deux modèles compréhensifs rendant compte de la réalité distincte des garçons et des filles quant à leur manière de s'inscrire dans le monde scolaire et quant aux stratégies qu'ils déploient pour s'y adapter. Ces deux modèles ne sont pas parfaitement étanches, tant s'en faut, mais ils permettent, croyons-nous, de mieux comprendre certaines différences entre les garçons et les filles, tout particulièrement chez ceux et celles qui sont à risque sur le plan de l'abandon et de l'échec scolaire, et, éventuellement, de mieux cibler nos interventions à l'endroit des garçons éprouvant des difficultés dans leur cheminement scolaire, personnel et social.

Jacques Roy

Techniques de travail social

Cégep de Sainte-Foy

Josée Bouchard

Techniques de travail social Cégep de Sainte-Foy

Marie-Anne Turcotte

Techniques de travail social Cégep de Sainte-Foy

\section{BIBLIOGRAPHIE}

Bajoit, G. (1997). «Qu'est-ce que le sujet? », dans G. Bajoit, F. Digneffe, J.-M. Jaspard et Q. N. de Brauwere (dir.), Jeunesse et société. La socialisation dans un monde en mutation, Bruxelles, De Boeck Université, coll. « Ouvertures sociologiques », p. 19-41.

Cherkaoui, M. (1990). «Socialisation », dans R. Boudon, P. Besnard, M. Cherkaoui et B.P. Lécuyer (dir.), Dictionnaire de la sociologie, Paris, Librairie Larousse, p. 181.

Chouinard, R., J.Bergeron, C. Vezeau et M. Janosz (2010). " Motivation et adaptation psychosociale des élèves du secondaire selon la localisation socioéconomique de leur école », Revue des sciences de l'éducation, vol. 36, n² 2, p. 321-342.

Dubar, C. (2005). «Socialisation », dans M. Borlandi, R. Boudon, M. Cherkaoui et B. Valade (dir.), Dictionnaire de la pensée sociologique, Paris, Presses universitaires de France, p. 647-650.

Dubar, C. (1995). La socialisation. Construction des identités sociales et professionnelles, $2^{\mathrm{e}}$ éd., Paris, Armand Colin.

Galland, O. (2007). Sociologie de la jeunesse, $4^{\mathrm{e}}$ éd., Paris, Armand Colin.

Lahire, B. (2004). La culture des individus : dissonances culturelles et distinction de soi, Paris, La Découverte.

Rivière, B., en collaboration avec J. Jacques (2002). Les jeunes et les représentations sociales de la réussite, Montréal, Éditions Logiques. 
Roy, J. (2011). Quête identitaire et réussite au cégep. Une étude de cas : la pratique d'activités parascolaires dans le réseau collégial, Québec, Presses de l'Université du Québec, coll. « Temps libre et culture ».

Roy, J. (2008). Entre la classe et les mcjobs. Portrait d'une génération de cégépiens, Québec, Les Presses de l'Université Laval, coll. "Regards sur la jeunesse du monde », série Analyses et essais.

Roy, J., J. Bouchard et M.-A. Turcotte, en collaboration avec G. Tremblay et S.-O. Fournier (2010). Filles et garçons au collégial : des univers parallèles? Étude sur la problématique des genres et la réussite scolaire, rapport de recherche PAREA, Québec, Cégep de Sainte-Foy, Équipe Masculinités et société et Observatoire Jeunes et société.

Roy, J., J. Bouchard et M.-A. Turcotte (2008). La conciliation entre le travail et les études chez les collégiens : un paradigme en évolution. Étude sur le travail rémunéré en milieu collégial, rapport de recherche PAREA, Québec, Cégep de Sainte-Foy et Observatoire Jeunes et société.

Sciolla, L. (2005). «Identité », dans M. Borlandi, R. Boudon, M. Cherkaoui et B. Valade (dir.), Dictionnaire de la pensée sociologique, Paris, Presses universitaires de France, p. 335-338.

Tremblay, G. et P. L'Heureux (2010). « La genèse de la construction de l'identité masculine », dans J.-M. Deslauriers, G. Tremblay, S. Genest Dufault, D. Blanchette et J.-Y. Gagné (dir.), Regards sur les hommes et les masculinités. Comprendre et intervenir, Québec, Presses de l'Université Laval, p. 91-123. 